Eco matemático ISSN: 1794-8231 (Impreso), E-ISSN: 2462-8794 (En línea) Volumen 8 (1) Enero-Diciembre de 2017, páginas 49-61

\title{
Fortalecimiento del pensamiento numérico a través de estrategias didácticas que desarrollen competencias comunicativas en los estudiantes del grado tercero de educación primaria
}

\section{Strengthening of numerical thinking through didactic strategies that develop communicative skills in students of the third grade of primary education}

\section{Fortalecimento do pensamento numérico por meio de estratégias didáticas que desenvolvem habilidades comunicativas em alunos do terceiro ano do ensino fundamental}

\author{
Diana del Pilar Iscalá-Tobito ${ }^{\mathrm{a}^{*}}$ \\ Licenciatura en matemáticas y computación, Institución Educativa Luis Carlos Galán Sarmiento. \\ Cúcuta, Colombia. Orcid: 0000-0003-4910-0996
}

Forma de citar: Iscalá, D. (2017). Fortalecimiento del pensamiento numérico a través de estrategias didácticas que desarrollen competencias comunicativas en los estudiantes del grado tercero de educación primaria. Eco matemático 8(1). 49-61

Recibido: agosto 2 de 2016

Aceptado: noviembre 7 de 2016

\section{Palabras clave}

Pensamiento numérico, Estrategias didácticas, Competencias Comunicativas, Educación Primaria.

\section{Keywords}

Numerical thinking, didactic strategies, communication skills, primary education.

\begin{abstract}
Resumen: El presente artículo titulado "Fortalecimiento del pensamiento numérico a través de estrategias didácticas que desarrollen competencias comunicativas en los estudiantes del grado tercero de educación primaria”, se refiere a una investigación que se encuentra en proceso, la cual surge de las experiencias pedagógicas vividas por su autora, quien ha venido observando las dificultades comunicativas que presentan los educandos de este grado y nivel de estudio, por lo cual considera pertinente diseñar diversas estrategias didácticas, que les permitirán no solamente fortalecer su pensamiento numérico, sino también facilitaran su proceso comunicacional que se proyectará en su vida diaria. Para el logro de este objetivo se propone una metodología de investigación acción, desde el enfoque cualitativo, donde el objeto de estudio es el proceso de aprendizaje de 14 estudiantes del grado tercero, de la jornada de la mañana de la Sede José Eusebio Caro No. 23, cuyas edades promedio está entre los 8 y 11 años. Se utilizan como instrumentos de recolección de información los resultados obtenidos en las pruebas SABER con su respectivo análisis, el conversatorio, la prueba diagnóstica, la observación directa, el diario pedagógico y la aplicación de talleres en clase.
\end{abstract}

\begin{abstract}
This article entitled "Strengthening numerical thinking through didactic strategies that develop communicative skills in students in the third grade of primary education", refers to a research that is in process, which arises from the pedagogical experiences experienced by its author, who has been observing the communicative difficulties presented by the students of this degree and level of study, for which it considers pertinent to design various teaching strategies, which will allow them not only to strengthen their numerical thinking, but also to facilitate their communicational process that will be projected in your daily life. To achieve this goal, an action research methodology is proposed, from the qualitative approach, where the object of study is the learning process of 14 students of the third grade, of the morning session of
\end{abstract}

\footnotetext{
* Autor para correspondencia dipiisto@hotmail.com

http://dx.doi.org/10.22463/17948231.1476
} 
the Headquarters José Eusebio Caro No. 23, whose average ages are between 8 and 11 years. The results obtained in the SABER tests with their respective analysis, the discussion, the diagnostic test, the direct observation, the pedagogical diary and the application of workshops in class are used as instruments for gathering information.

\section{Palavras-chave}

Pensamento numérico, estratégias didáticas, habilidades de comunicação, ensino fundamental.
Resumo: Este artigo intitulado "Reforçar o pensamento numérico através de estratégias de ensino para desenvolver habilidades de comunicação em estudantes de nível educacional terceiro grau" refere-se a uma investigação que está em curso, que decorre a partir das experiências educacionais de sua autor, que tem sido observando as dificuldades de comunicação apresentadas pelos estudantes deste grau e nível de estudo, e, portanto, considera adequado para projetar várias estratégias de ensino que lhes permita não só para reforçar o seu pensamento numérico, mas também facilitar o seu processo de comunicação para ser exibido em sua vida diária. Para atingir este objectivo uma pesquisa metodologia proposta ação, a partir da abordagem qualitativa, onde o objeto de estudo é o processo de aprendizagem de 14 alunos da terceira série do dia na Sede da manhã José Eusebio Caro Não. 23, cujas idades médias estão entre 8 e 11 anos. Eles são usados como instrumentos de coleta de dados, os resultados dos testes para saber sua análise, o grupo de discussão, o teste de diagnóstico, observação direta, as oficinas educativas diárias e aplicação em sala de aula.

\section{Introducción}

El docente debe asumir el reto de contribuir a que el proceso de enseñanza se inserte dentro del nuevo paradigma que propone el Ministerio de Educación en su Currículo Básico Nacional Colombiano, donde los protagonistas son los estudiantes, de tal forma que éstos tengan capacidad para desarrollar su pensamiento lógico, la convivencia social y así estén preparados para enfrentar los desafíos de una sociedad que presenta cambios constantes y exige de ellos capacidad de análisis en la toma de decisiones.

En el proceso de aprendizaje juega un papel importante el manejo de recursos utilizados por el educador, éstos facilitan el desarrollo de habilidades y destrezas y la interacción con el medio, permite generar nuevas experiencias tales como explorar, probar, reformular hipótesis, representar de manera concreta conceptos abstractos e integrar las distintas áreas académicas de resolución de problemas en Matemáticas.

El docente formado en nuevas técnicas y estrategias didácticas es un docente capaz de asumir todos los retos que impone la vida moderna y se encarga de ir cerrando la "brecha del siglo XXI" que aún existe en la educación Colombiana, de poder interactuar con sus estudiantes generando nuevos escenarios formativos a través de la comunicación verbal, de desarrollar habilidades para la búsqueda y selección de la información, permitiendo así un aprendizaje más significativo, cooperativo y de mayor comunicación entre profesor y estudiante, con un alto grado de interdisciplinariedad.

La presente investigación parte del análisis de las dificultades que presentan los estudiantes del grado tercero de la sede José Eusebio Caro No. 23, al momento de expresar soluciones a un enunciado matemático, interpretar gráficos, comunicar sus propias soluciones y evaluar y aceptar las opiniones de sus demás compañeros. Estas falencias se evidencian en los resultados de las pruebas SABER de años anteriores, que expide el Instituto Colombiano para el Fomento de la Educación Superior (ICFES), al igual que en los resultados obtenidos en las pruebas internas realizadas en la institución.

La propuesta de investigación planteada se basa en el fortalecimiento de la competencia comunicativa del área de matemáticas en los estudiantes del grado 
tercero del colegio Luis Carlos Galán Sarmiento Sede José Eusebio Caro No. 23 mediante la aplicación de una estrategia didáctica que facilite al estudiante su aprendizaje de forma eficaz y a la vez que sea un apoyo para el docente al realizar sus prácticas pedagógicas, logrando de esta forma mejorar el rendimiento en el área de matemáticas

Dicha propuesta nace de la dificultad que presentan los estudiantes al momento de expresar en forma escrita, oral y visual soluciones a enunciados matemáticos planteados durante las actividades desarrolladas en clase, se evidencia una gran problemática que impide el progreso en cada uno de ellos en esta área de conocimiento. Al mismo tiempo la necesidad de fortalecer la comunicación como uno de los procesos más importantes para resolver problemas, interactuar con sus compañeros y docentes y expresar sus inquietudes y sus opiniones al momento del trabajo en el aula.

Al hacer una revisión del Proyecto Educativo de la institución se observa que el área de matemáticas se encuentra contemplada en el plan de estudios y es impartida desde el grado transición (dimensión) hasta el grado once, con una intensidad horaria de cinco horas semanales en la básica primaria, cuatro en la básica secundaria y tres en la media técnica.

La institución se enmarca en la aplicación del modelo pedagógico cognitivo constructivo el cual hace énfasis en que el estudiante es quien construye su propio conocimiento y despierta su espíritu investigador ya que se siente motivación por aprender por sí solo; en este modelo el docente cumple el papel de facilitar y estimular experiencias en un ambiente de aprendizaje continuo dentro del aula de clase. El modelo cognitivo constructivo avanza en forma gradual y posee estructuras que están diferenciadas en un orden jerárquico.

Dentro de los teóricos que soportan el modelo cognitivo constructivo se encuentra Piaget. Para Piaget (1950) el niño aprende a través del hacer y el explorar, comprende su entorno para luego hacer una comparación de lo que ya sabe y lo que descubre. Este autor se centra en los procesos mentales del estudiante y principalmente en la capacidad que posee para avanzar hacia las habilidades cognitivas cada más compleja, ya sea que el mismo lo haga o que lo logre con la ayuda del docente. Según Piaget (ob. cit.), el desarrollo cognitivo del niño es secuencial y se da en cuatro estadios o etapas que son: sensoria motora que va hasta los dos años, preoperacional hasta aproximadamente los 7 años, operaciones concretas hasta los 11 años y las operaciones formales la cual se extiende hasta la edad adulta.

Por otra parte, Vygotsky manifiesta que los procesos mentales del niño tienen un origen social, que todo niño nace con habilidades mentales y a medida que interactúa con sus compañeros estas habilidades se convierten en funciones mentales superiores, entre más el niño se relacione su conocimiento aumenta. Sostiene que los niños desarrollan su aprendizaje al interactuar y compartir con otros niños, propone al lenguaje como herramienta mediadora del desarrollo y manifiesta que el niño no solo utiliza el lenguaje para comunicarse sino para organiza, planear y guiar su propio comportamiento, el diálogo interno convierte al individuo en seres más comunicativos socialmente.

Existen diversas estrategias de enseñanza para aplicar la teoría de Vygotsky, entre esas se encuentran: ofrecer ayuda al estudiante cuando sea necesario, motivarlo y apreciar sus esfuerzos, emplear el aprendizaje colaborativo y animarlo a utilizar el habla privada que es primordial en el desarrollo del pensamiento.

Respeto al enfoque que maneja la institución se encuentra el aprendizaje significativo que hace referencia a que los nuevos conocimientos que se adquieren están ligados con los anteriores, su mayor exponente es Ausubel quien manifiesta que la enseñanza es un proceso que permite que el estudiante aumente y sobre todo perfeccione el conocimiento que ya tiene. Para Ausubel (1973) el niño aprende significativamente cuando relaciona los nuevos conocimientos con los que ya posee, el elemento primordial del aprendizaje significativo 
es cómo la nueva información se integra en la estructura de conocimientos a lo largo del tiempo. Por otra parte, la actitud juega un papel primordial ya que dependiendo de la predisposición que tenga el estudiante al momento de aprender se sentirá motivado por adquirir nuevos conocimientos.

\section{Materiales y método}

La presente investigación se encuentra enmarcada dentro de un enfoque cualitativo que se caracteriza por ser una metodología cuyo centro es el sujeto. Su base es la constante observación y el análisis de situaciones que tienen relación con el comportamiento entre las personas. Blasco y Pérez (2007), señalan que "La investigación cualitativa estudia la realidad en su contexto natural y cómo sucede, sacando e interpretando fenómenos de acuerdo con las personas implicadas. Utiliza variedad de instrumentos para recoger información como las entrevistas, imágenes, observaciones, historias de vida, en los que se describen las rutinas y las situaciones problemáticas, así como los significados en la vida de los participantes. Toda investigación cualitativa se basa en la observación y construye el conocimiento teniendo en cuenta el comportamiento del individuo y su conducta" (p.25)

Por otra parte, en esta investigación se retoma la investigación acción con el propósito de mejorar y comprender la práctica educativa convirtiendo así a los estudiantes en entes investigadores. Elliot (2000) la define como "Un estudio de una situación social con el fin de mejorar la calidad de la acción dentro de la misma" (p.24), Lomax (1990) define la investigación acción como "Una investigación e la práctica profesional con la intensión de ocasionar una mejora" (p.4) y Kemis (1984), "una forma de indagación autorreflexiva realizado por quienes participan en situaciones sociales para mejorar la racionalidad y la justicia de: a) sus propias prácticas sociales o educativas, b) su comprensión sobre las mismas y c) las situaciones e instituciones en que estas prácticas se realizan”.(p.4)

Y finalizando con Murillo F. en palabras de Kemmis y McTaggart, "Los principales beneficios de la investigación-acción son: La mejora de la práctica, la comprensión de la práctica y la mejora de la situación en la que tiene lugar la práctica. La investigación acción se propone mejorar la educación a través del cambio y aprender a partir de las consecuencias de los cambios". (p. 6)

\section{Resultados y análisis}

Al analizar los resultados de las pruebas externas (SABER) del año 2017, se centra la atención en los resultados obtenidos en el área de matemáticas específicamente en el grado tercero. La gráfica 1 muestra el porcentaje de estudiantes en cada nivel



Gráfica 1. Porcentaje de estudiantes por niveles de desempeño. Matemáticas $3^{\circ}$ - 2017. Fuente: Prueba SABER del año 2017. 
de desempeño, cada uno hace referencia a una descripción cualitativa sobre lo que el estudiante es capaz de hacer cuando se enfrenta a preguntas de distintos rangos de dificultad en una situación de contexto específico. Se evidencia claramente que el nivel INSUFICIENTE tiene un $26 \%$, el nivel MÍNIMO 43\%, el nivel SATISFACTORIO $24 \%$ y el nivel AVANZANDO un $7 \%$, siendo este el de menor porcentaje.

En cuanto a los resultados obtenidos en la competencia comunicación, la cual hace referencia a la capacidad del estudiante para expresar ideas, usar diferentes tipos de representación, describir relaciones matemáticas, escribir situaciones o problemas usando el lenguaje escrito, pictórico y gráfico, se evidencia dificultad. En la gráfica 2 se observa que en la competencia comunicación el establecimiento presenta debilidad en comparación con los establecimientos que presentan un puntaje promedio similar al área y grado.

En cuanto a los componentes evaluados en las pruebas, la gráfica 3 muestra los resultados obtenidos a partir de la comparación del desempeño del establecimiento con el desempeño de los establecimientos del país que obtuvieron un puntaje promedio similar. Se evidencia que el pensamiento numérico variacional presenta debilidad.

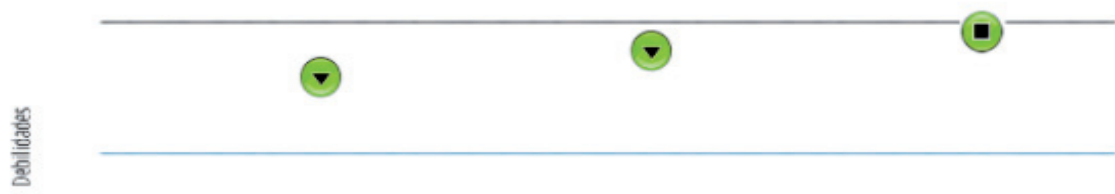

\begin{tabular}{lll}
\hline Razonamiento & Comunicación
\end{tabular}

Gráfica 2. Resultados Pruebas SABER. - Competencia Comunicación-Matemáticas $3^{\circ}$.

Fuente: Prueba SABER del año 2017.

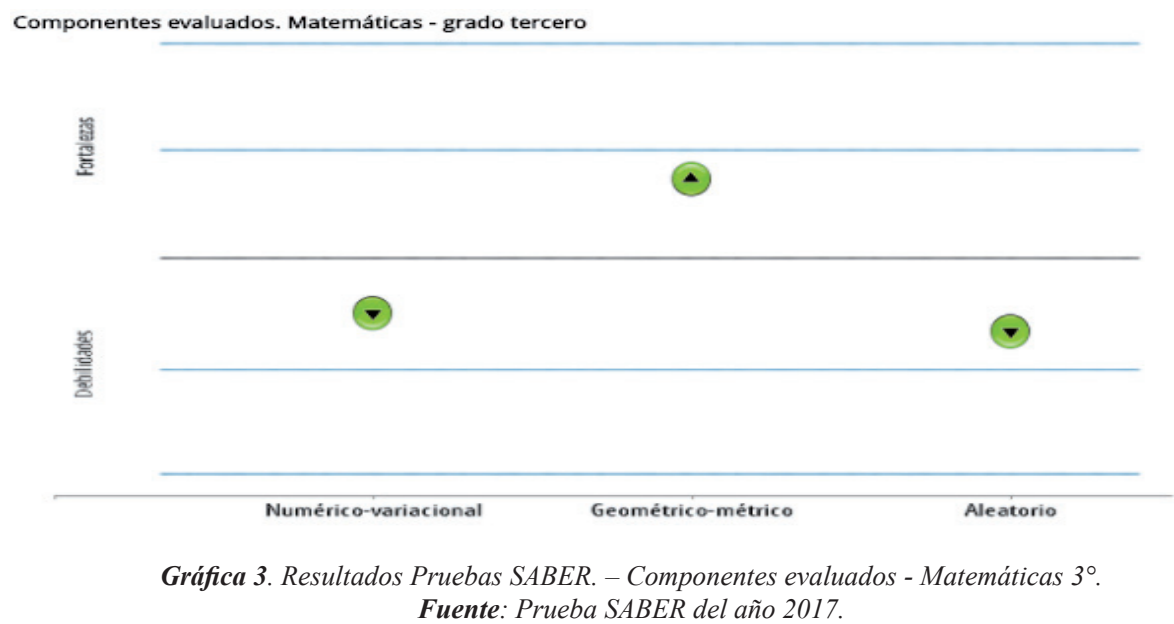

Eco matemático ISSN: 1794-8231 (Impreso), E-ISSN: 2462-8794 (En línea) Volumen 8 (1) Enero-Diciembre de 2017, páginas 49-61 
Al observar los resultados obtenidos en las Pruebas Saber, se puede identificar que los niveles satisfactorio $\mathrm{y}$ avanzado tienen un porcentaje bajo, el propósito esperado es elevar estos resultados y al mismo tiempo fortalecer la competencia comunicación mediante la aplicación de la estrategia que estimule al estudiante a pesar y comunicar en forma oral y escrita empleando un vocabulario acorde a su nivel.

El Índice Sintético de Calidad Educativa (ISCE), busca medir el estado de la calidad de los colegios en una escala de 1 a 10 en cuatro factores: progreso, desempeño, eficiencia y ambiente escolar. En cuanto al progreso se evidencia que la institución ha mejorado, el desempeño es la comparación de los resultados con relación a otras instituciones educativas, la eficiencia mide la cantidad de estudiantes promovidos y el ambiente escolar establece o evalúa el acompañamiento educativo y el ambiente en el aula, a su vez dichos resultados se comparan con los M.M.A. que es la meta de mejoramiento anual, es decir, cuanto debe mejorar de acuerdo a los resultados obtenidos.

Los resultados del Índice Sintético de Calidad Educativa (ISCE) se muestran en la siguiente gráfica:
Esta propuesta es pensada inicialmente como la posibilidad de relacionar los diferentes usos lingüísticos y comunicativos en matemáticas para comprender y facilitar los procesos de enseñanza y aprendizaje del área. El estudio se orienta a lograr que el docente mejore su práctica pedagógica en el área de matemáticas y fortalezca la competencia comunicativa de los estudiantes de tercer grado dejando de lado la educación tradicional, las orientaciones magistrales y acumulativas de conocimientos inconexos con la realidad, por una forma más amena y práctica. Esto constituye una novedosa praxis de la enseñanza, teniendo este estudio relevancia social pues sirve como apoyo para que el docente desarrolle en sus estudiantes una de las competencias básicas que implica aprender matemáticas, destacando en este caso la competencia comunicativa que busca que él sea capaz de interpretar y a su vez argumentar la solución a situaciones que se le planteen.

Un estudiante competente en matemáticas es aquel que posee la capacidad para ejecutar tareas matemáticas, comprender y argumentar posibles soluciones. Para lograr ser competente se requiere de tiempo ya que este es un proceso continuo que a lo largo de la vida escolar se mejora y se

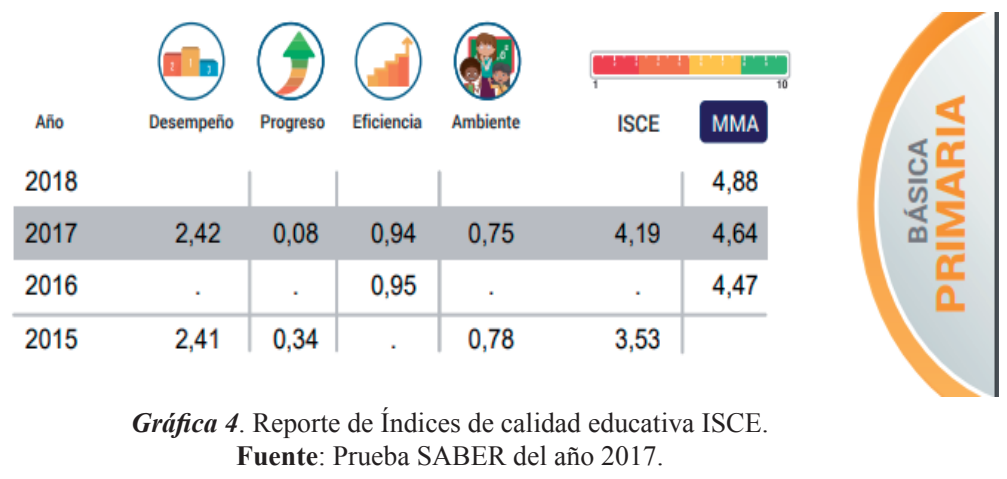

Al observar los resultados de los cuatro factores el promedio obtenido es de 4,19 que está por debajo de la meta de mejoramiento anual que se encuentra en 4,64, es decir que no se supera de acuerdo a los resultados del año 2017. perfecciona. Las competencias en matemáticas permiten que el estudiante adquiera habilidades que le permitan aplicar los conocimientos que posee en diferentes contextos. Dentro de las competencias trabajadas en esta área se encuentra la 
competencia comunicativa, en dicha competencia el estudiante desarrolla habilidades para expresarse transmitiendo información plasmada en una situación con sus posibles soluciones.

En la práctica pedagógica se ha encontrado dificultades en los estudiantes del grado tercero de la Sede José Eusebio Caro No. 23 en cuanto al rendimiento en el área de matemáticas. Se parte de la falta de motivación del estudiante por aprender las matemáticas, considerada ésta como algo difícil de entender y de gran complejidad, reflejada en la apatía al momento de realizar las actividades planteadas en el aula de clase. Por otro lado, se aprecia dificultad en el proceso lecto escritor. La mayoría de estudiantes no interpretan ni resuelven enunciados matemáticos, manejan poco vocabulario y esto trae inconvenientes al plasmar en forma escrita sus ideas y posibles soluciones a ejercicios que se plantean. De igual forma la expresión verbal presenta deficiencia, hay inconvenientes al expresar a los demás compañeros sus ideas al igual que al docente, al no comprender lo que lee no es capaz de transmitir a los demás lo leído. Estas dificultades repercuten notoriamente cuando el docente plantea la actividad a desarrollar en clase, al leer con dificultad y no escribir coordinando sus ideas, el estudiante poco analiza e interpreta y escasamente logra desarrollar las actividades propuestas en un taller o guía de trabajo y cuando se hace necesario que se exprese ante sus compañeros para compartir sus ideas y comunicar resultados obtenidos se encuentran fallas notorias. Algo similar sucede a la hora de realizar una actividad asignada, el estudiante opta por abandonarla o por dar respuestas incoherentes tanto en forma oral o escrita.

Desde esta observación diagnóstica se requiere realizar una investigación que permita fortalecer la competencia comunicativa propiciando una estrategia aplicada desde el pensamiento numérico, la cual tendrá como resultados el mejoramiento en las pruebas saber, así como en las evaluaciones internas. Mediante la aplicación de la estrategia didáctica en las prácticas educativas de matemáticas se logra que el estudiante fortalezca la competencia comunicación y pueda lograr un aprendizaje significativo que se verá reflejado en los próximos resultados de las pruebas saber.

La presente investigación, está sustentada en teorías relevantes fundamentadas en definiciones de varios autores, ligadas con la temática del presente estudio.

A continuación, se presentan las bases teóricas que sustentan la investigación:

\section{Aprendizaje}

El aprendizaje es el proceso de adquisición de conocimientos, habilidades, valores y actitudes, posibilitado mediante el estudio, la enseñanza o la experiencia. En este estudio investigativo se toma como referencia las diferentes teorías de Piaget, Vygotsky y Ausubel ya que el modelo pedagógico de la institución, objeto de estudio es el cognitivoconstructivo y el enfoque es el aprendizaje significativo.

Al respecto Dongo, A. (2008), afirma que "Todo nuevo cambio o modificación de los esquemas en función de la experiencia es conceptuada como aprendizaje" (p.171), es decir que para que se lleven a cabo procesos de aprendizaje es necesario un cambio en la conducta, las actitudes y en la asimilación de conocimientos e información dentro y fuera del contexto educativo.

Para Piaget "El aprendizaje alcanza un significado adaptativo en la medida en que expresa una acomodación activa por parte del sujeto y progresa, al buscar la novedad exterior, en función de las coordinaciones de los esquemas de inteligencia" Dongo, A. (ob. cit.), es decir que el ser humano aprende de acuerdo a la edad de maduración e incorpora los nuevos conocimientos a partir de las experiencias dadas, de acuerdo a los estadios en que se encuentra teniendo en cuenta que cada una tiene características especiales y que el ser humano interactúa con el medio para la construcción del conocimiento. 
Según (Kammi, 1973), citado por Rodríguez Arocho, (1999), manifiesta que en la pedagogía piagetiana es imposible avanzar el entendimiento de la persona simplemente comunicándole información. La buena pedagogía debe implicar la presentación de situaciones para que el niño y la niña experimenten; es decir, realicen actividades con la intención de ver qué ocurre, manipulen símbolos, formulen preguntas y busquen sus propias respuestas, reconcilien lo que encuentran una vez con lo que encuentran en otras ocasiones, y comparen y discutan sus hallazgos con los de sus compañeros y compañeras. En consecuencia, el estudiante aprende a partir de la interiorización de un proceso continuo de exploración, asimilación y acomodación en donde debe interactuar con el nuevo conocimiento para apropiarse de él.

Por suparte Vygotsky proponelateoría sociocultural o socio-histórica del aprendizaje en donde el entorno, es decir la cultura y la sociedad influyen en la adquisición del conocimiento, en el desarrollo cognitivo y en el aprendizaje del ser humano, ya que este interactúa con el ambiente que lo rodea. Para Baquero, R. (1996). Vygotsky manifiesta que "Desde este punto de vista aprendizaje no equivale a desarrollo; no obstante, el aprendizaje organizado se convierte en desarrollo mental y pone en marcha una serie de procesos evolutivos que no podrían darse nunca al margen del aprendizaje. Así pues, el aprendizaje es un aspecto universal y necesario del proceso de desarrollo culturalmente organizado y específicamente humano de las funciones psicológicas" (p.3). En este sentido la interacción social es fundamental en el desarrollo de las habilidades y de ahí se potencializa el papel del docente como facilitador o mediador de los procesos en el aula, quien lidera y motiva los avances en los procesos dados proporcionando las herramientas necesarias para que el estudiante avance en los procesos cognitivos. Para este autor, la enseñanza y la educación constituyen formas universales del desarrollo psíquico de la persona y el instrumento esencial de enculturación $\mathrm{y}$ humanización.
Matos, (1996), citado por Chaves Salas, (2001), expone que para Vygotsky el aprendizaje precede al desarrollo. Entre el aprendizaje y el desarrollo existe una relación de tipo dialéctica. Una enseñanza adecuada contribuye a crear zonas de desarrollo próximo; "es decir va a servir de imán para hacer que el nivel potencial de desarrollo del educando se integre con el actual. Estas modificaciones, a su vez pueden promover progresos en el desarrollo cognoscitivo general. Como han señalado algunos, la ZDP es un diálogo entre el niño y su futuro, entre lo que es capaz de hacer hoy y lo que será capaz de hacer mañana y no entre el niño y su pasado" (p.62). En consecuencia, el ser humano inicia su proceso de aprendizaje al relacionarse con todo lo que lo rodea, es decir el medio exterior, el cual le proporciona la información necesaria para construir su saber, en forma individual y colectiva.

Ausubel propone el aprendizaje significativo el cual centra su mayor interés en relacionar la información nueva con los preconceptos de los estudiantes. Para que dicho proceso se realice es necesario que haya un significado, y se encajen los conocimientos de modo que se complementen. Según este teórico "el aprendizaje significativo ocurre cuando una nueva información "se conecta" con un concepto relevante ("subsunsor") pre existente en la estructura cognitiva, esto implica que, las nuevas ideas, conceptos y proposiciones pueden ser aprendidos significativamente en la medida en que otras ideas, conceptos o proposiciones relevantes estén adecuadamente claras y disponibles en la estructura cognitiva del individuo y que funcionen como un punto de “anclaje" a las primeras."(p.2)

\section{Pensamiento Numérico}

McIntosh, (1992) citado por Obando, G., \& Vásquez, N. (2008) afirma que el pensamiento numérico se refiere a la comprensión en general que tiene una persona sobre los números y las operaciones junto con la habilidad y la inclinación a usar esta comprensión en formas flexibles para hacer juicios matemáticos y para desarrollar estrategias útiles al manejar números y operaciones, 
es decir el pensamiento numérico hace referencia a la habilidad que posee el ser humano para emplear los números en diferentes situaciones cotidianas dando soluciones acertadas a cada una de ellas.

Encarna Castro (2008), citado por Saldaña, (2012) señala que el pensamiento numérico trata de aquello que la mente puede hacer con los números, y que está presente en todas aquellas actuaciones que realizan los seres humanos relacionadas con los números. Asimismo, nos recuerda que las investigaciones llevadas a cabo dentro de este campo ponen el énfasis en los procesos cognitivos de los sujetos, y en ellas se contemplan, entre otros aspectos, los siguientes:

- La naturaleza y características de los aprendizajes numéricos, así como los errores y dificultades que se presentan en dichos procesos

- Las semejanzas y diferencias en la construcción de los conocimientos por parte de diferentes individuos

- Las componentes culturales, que influyen, tanto en la construcción de los conocimientos como en los modos de abordar la enseñanza de los mismos.

Por consiguiente, es importante desarrollar el pensamiento numérico en los niños desde temprana edad ya que esto les permite afianzar habilidades matemáticas a partir del conocimiento y uso de los números tanto en el conteo, numeración, manejo de operaciones, resolución de problemas, entre otros.

Según el Ministerio de Educación Nacional en el documento Lineamientos Curriculares para el área de matemáticas establece que "El pensamiento numérico se adquiere gradualmente $\mathrm{y}$ va evolucionando en la medida en que los alumnos tienen la oportunidad de pensar en los números y de usarlos en contextos significativos, y se manifiesta de diversas maneras de acuerdo con el desarrollo del pensamiento matemático"(p.26), de esta manera a medida que el estudiante explora, conoce y emplea los números, avanza en procesos matemáticos cada vez más complejos, haciendo uso de ellos en situaciones que se le presenten.

\section{Estrategia Didáctica}

Feo, R. (2010) define las estrategias didácticas como "los procedimientos (métodos, técnicas, actividades) por los cuales el docente y los estudiantes, organizan las acciones de manera consciente para construir y lograr metas previstas e imprevistas en el proceso enseñanza y aprendizaje, adaptándose a las necesidades de los participantes de manera significativa" (p.222). Es decir que a partir de la planeación de los momentos pedagógicos el docente plantea las actividades precisas, adecuadas y necesarias para que el estudiante pueda participar y hacer construcción del aprendizaje.

Mosquera y Velasco (2010) exponen que "Al entender que la estrategia didáctica es el conjunto de procedimientos, apoyados en técnicas de enseñanza, que tienen por objeto llevar a buen término la acción pedagógica del docente, se necesita orientar el concepto de técnica como procedimientos didácticos y el recurso particular para llevar a efecto los propósitos planeados desde la estrategia. Las estrategias didácticas apuntan a fomentar procesos de autoaprendizaje, aprendizaje interactivo y aprendizaje colaborativo. (p.3), por lo tanto, es necesario conocer el contexto, los recursos y las expectativas de los educandos para la planeación y aplicación de las estrategias didácticas más acertadas de acuerdo a la edad y al nivel cognitivo teniendo en cuenta el modelo pedagógico y el enfoque de la institución objeto de estudio.

Castañeda, M. (2013) expone que "Es sustancial, plantear estrategias didácticas que contemplen los objetivos de Enseñanza-Aprendizaje a partir de los diversos métodos, los cuáles deben dirigirse a las necesidades particulares de cada asignatura" (p.2), a partir de esto se hace necesario la selección adecuada de las estrategias que se aplicarán en los diferentes momentos pedagógicos. 
Para Feo (ob. cit.) se puede llegar a una clasificación de estos procedimientos, según el agente que lo lleva a cabo, de la manera siguiente: (a) estrategias de enseñanza; (b) estrategias instruccionales; (c) estrategias de aprendizaje; y (d) estrategias de evaluación. (p.222) comportamientos que ciertas personas poseen en mayor medida que otras y que las transforman en más eficaces para una situación dada. Las competencias representan un rasgo de unión entre las características individuales y las cualidades requeridas para conducir las misiones profesionales

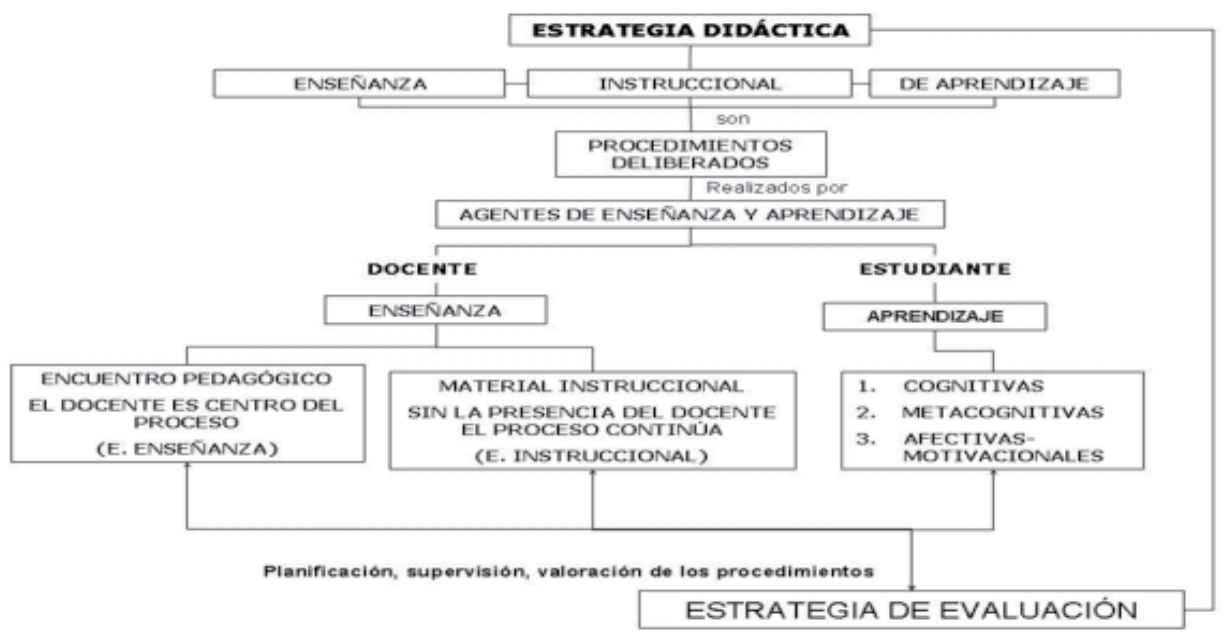

Gráfica 5. La estrategia didáctica, su clasificación.

Fuente: Feo (2010).

En el quehacer docente se inicia el proceso con estrategias de enseñanza donde a partir de un diálogo dirigido se establecen las pautas para que el estudiante exprese sus expectativas acerca de la actividad a desarrollar; luego se aplican las estrategias instruccionales en las cuales el docente facilita el material impreso para que el estudiante desarrolle las actividades planteadas, en este momento el docente brinda acompañamiento si el estudiante lo requiere o emplea otros recursos adicionales. Seguidamente se dan las estrategias de aprendizaje donde el estudiante aplica las técnicas de estudio y reconoce el uso de habilidades cognitivas que le permitan llevar a cabo el proceso de aprendizaje, finalmente se aplican las estrategias de evaluación donde se verifica la efectividad, los aciertos y las dificultades del proceso realizado.

\section{Competencia Comunicativa}

Levy-Leboyer citado por Charria Ortiz (2011) señala que "las competencias son una lista de prefijadas" (p. 58), es decir, un estudiante es competente en la medida que desarrolla su saber conocer, su saber hacer y su saber ser en el contexto donde se desenvuelve generando nuevos conocimientos y habilidades que le permitan desempeñarse en cualquier ambiente o situación.

Para el Ministerio de Educación Nacional (MEN), en su documento Estándares Básicos de Competencias expone que "Las competencias se desarrollan a lo largo de la vida, y es función del sistema educativo aportar a su desarrollo para alcanzar la calidad deseada contando, con criterios claros y públicos que permitan establecer si se están alcanzando o no los niveles que como sociedad nos hemos propuesto". (p.15), por consiguiente, el sistema educativo debe apuntar a formar estudiantes competentes en todas las áreas de acuerdo a los estándares de calidad propuestos por cada país. 
En cuanto a la competencia comunicativa, ésta hace referencia a la habilidad que tiene el estudiante para emitir juicios, argumentar, exponer, y hacer uso del lenguaje en los diferentes ámbitos. Para Hymes (2003) "La competencia comunicativa es el término más general para la capacidad comunicativa de una persona, capacidad que abarca tanto el conocimiento de la lengua como la habilidad para utilizarla. La adquisición de tal competencia está mediada por la experiencia social, las necesidades y motivaciones, y la acción, que es a la vez una fuente renovada de motivaciones, necesidades y experiencias". (p.1) Esta competencia posibilita la participación apropiada del ser humano en diversas situaciones comunicativas.

Cenoz Iragui, (2004) afirma que "El concepto de competencia comunicativa propuesto por Hymes tiene gran fuerza como herramienta organizadora en las ciencias sociales y es utilizado con gran frecuencia en la lingüística y psicolingüística, especialmente en relación con la adquisición de la primera y la segunda lengua. Sin embargo, son muchos los investigadores que han complementado algunos aspectos de la definición de competencia comunicativa". (p.2). De ahí la importancia de fortalecer los procesos de desarrollo de la competencia comunicativa en todas las áreas del conocimiento, en especial el área de matemáticas donde el estudiante a partir de la comprensión e interpretación del lenguaje avanza en procesos de decodificación para el análisis de situaciones matemáticas relacionadas al pensamiento numérico.

Dicho autor expreso que la competencia comunicativasesubdivideenotrassubcompetencias (lingüística o gramatical, sociolingüística, social, discursiva o textual, pragmática o referencial, semiológica, funcional, literaria, estratégica), las cuales contribuyen a delimitarla mostrando su complejidad y extensión. De esta manera lo esquematiza la siguiente gráfica:

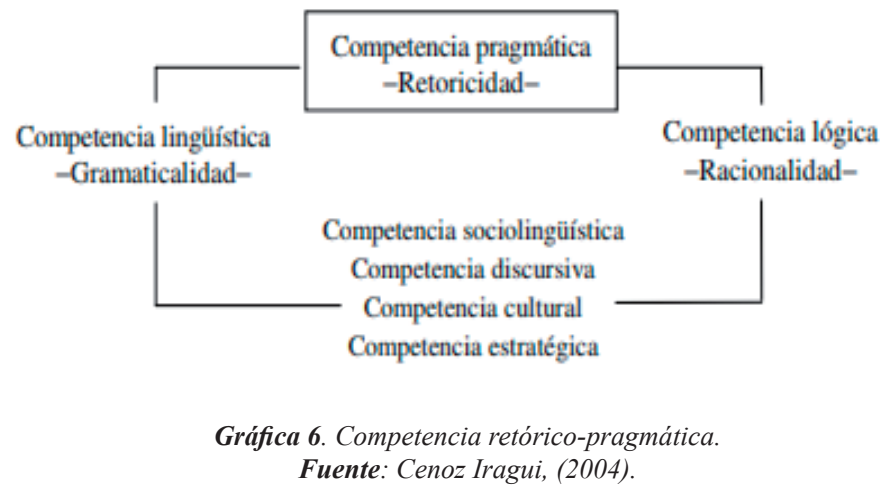

A través de las tres operaciones de gramaticalidad, retoricidad y racionalidad, actúan las competencias involucradas en la interacción comunicativa.

Guerrero, L. C. (2007), considera que "La competencia retórico-pragmática la entendemos como la competencia comunicativa que abarca a todas las demás, y donde opera la «retoricidad». Entendiendo por ésta, esa operación pragmática que activa a las demás competencias para adaptarse a la situación y lograr el objetivo comunicativo. En la retoricidad no operan sólo principios discursivos, sino que operan, también, reglas gramaticales, operaciones lógicas, y operaciones enciclopédicas en una relación de interdependencia para negociar el significado y lograr la comunicación, la puesta en común. Es decir que la retórica activa todas las subcompetencias para lograr procesos comunicativos que permitan que el estudiante exprese, manifieste y comparta sus resultados matemáticos justificando el desarrollo de la metodología realizada.

\section{Educación Primaria}

La Educación Primaria en Colombia, es el nivel en el cual, el niño de aproximadamente 5 o 6 años, empieza a recibir los conocimientos necesarios para desenvolverse en su medio, este nivel va hasta el grado quinto, más o menos 9 a 10 años de edad, donde el estudiante ha madurado en $\mathrm{su}$ formación integral y posee conocimientos, habilidades y destrezas en todas las áreas de estudio. Según el Artículo 21 de la ley 115 "la educación básica primaria corresponde al ciclo 
de los cinco (5) primeros grados de la educación básica". Esta etapa es importante para el niño no solo en su desarrollo cognitivo sino social ya que debe interactuar con sus semejantes y aprender a leer, a escribir, a comprender, a realizar diferentes operaciones matemáticas básicas, a desarrollar su motricidad fina y gruesa y a incorporar diferentes valores para relacionarse con los otros.

Según la Organización de Estados Iberoamericanos para la Educación, la Ciencia y la Cultura (OEI) en los Sistemas Educativos Nacionales de Colombia "La Educación Básica Primaria desarrolla planes y programas propios, integrando las áreas de conocimiento y formación en los tres primeros grados. La orientación y metodología varía de acuerdo con la población según sea rural o urbana" (p.2). Es deber de los docentes encargados de enseñar en el nivel de primaria, brindar las estrategias necesarias para lograr en sus educandos el desarrollo de todas sus potencialidades a su vez que se debe adaptar el currículo a los contextos, características y necesidades de los niños que pertenecen que están dentro de este rango de formación.

En cuanto a la educación primaria, es necesario definir y mencionar el artículo 21 de la Ley 115 de 1994, que establece los objetivos específicos de la educación básica en el ciclo de primaria. Es aquí donde el niño adquiere las bases que le van a permitir desenvolverse en diferentes contextos y avanzar en su formación para ser un ciudadano integral que se puede desarrollar en la sociedad.

\section{Conclusiones}

Durante la realización de una observación intragrupo, donde se llevó a cabo un conversatorio, una prueba diagnóstica y posteriormente 12 talleres organizados en tres sesiones de trabajo, se puede concluir inicialmente que se evidenció que durante el conversatorio los estudiantes demuestran timidez al momento de expresarse ante sus compañeros, el tono de la voz es demasiado bajo, lo que ocasiona que la mayoría se distraiga y pierda el interés por los aportes que realiza el compañero, las respuestas dadas a los interrogantes planteados son elementales, pues se nota el poco de manejo de vocabulario y les cuesta mucho trabajo realizar las justificaciones respectivas.

En la prueba diagnóstica se evidenció que los estudiantes presentaron dificultad en la lectura e interpretación de los enunciados ya que pedían asesoría al preguntar lo que debía hacer en cada ítem, se notó deficiencias en la escritura pues hubo respuestas con ideas incompletas, las justificaciones dadas en forma escrita fueron en su mayoría confusas pues no expresaba en forma clara la idea que se quería comunicar.

En cuanto a la realización de los talleres se notó la participación de los estudiantes en la realización de las actividades ya que lo hicieron con agrado pues manifestaron que les gusta llevar a cabo actividades lúdicas que contengan pasatiempos y otras actividades. El trabajo en equipo los motivó e interactuaron con facilidad con los demás compañeros. Al realizar la puesta en común y dar respuestas en forma verbal cabe destacar que el tono de voz debe ser alto para así poder concentrar la atención de los demás compañeros y evitar distracciones.

Finalmente se puede concluir que dichas actividades diseñadas permiten fortalecer la competencia comunicativa en matemáticas basada en el pensamiento numérico, con el fin de que los estudiantes desarrollen sus habilidades para interpretar, justificar $\mathrm{y}$ argumentan procesos $\mathrm{y}$ enunciados matemáticos mediante la participación individual y el trabajo cooperativo en el aula. Por consiguiente, dicha estrategia aplicada permitió fortalecer los procesos del pensamiento relacionados a la interpretación, argumentación y justificación viéndose reflejado el interés por comunicar en forma oral y escrita los pasos llevados a cabo al solucionar situaciones matemáticas planteadas y en las socializaciones dar aportes a las ideas que expresan los demás compañeros. La puesta en práctica del trabajo en equipo permitió comprobar que los estudiantes al interactuar con 
los compañeros se interesan más por las actividades logrando así aprendizajes significativos.

\section{Referencias}

Arias, F. G. (2012). El Proyecto de Investigación. Introducción a la metodología científica. 5ta. Fidias G. Arias Odón.

Ausubel, D. (1983). Teoría del aprendizaje significativo. Fascículos de CEIF, 1, 1-10.

Baquero, R. (1996). Vygotsky y el aprendizaje escolar (Vol. 4). Buenos Aires: Aique. (p.3).

Blasco Mira, J. E., \& Pérez Turpin, J. A. (2007). Metodologías de investigación en educación física y deportes: ampliando horizontes. Editorial Club Universitario.

Castañeda, M. (2013). Manual de estrategias didácticas.

Charria, V. (2011). Definición y clasificación teórica de las competencias y clasificación de las competencias académicas, profesionales y laborales, Las competencias del Psicólogo en Colombia.

Chaves Salas, A. L. (2001). Implicaciones educativas de la teoría sociocultural de Vygotsky. Educación, 25(2). (p.62)

Cenoz Iragui, J. (2004). El concepto de competencia comunicativa. J. Sánchez Lobato \& I. Santos.

Dongo, A. (2008). La teoría del aprendizaje de Piaget y sus consecuencias para la praxis educativa. Revista de investigación en psicología, 11(1), 167-181.

Elliot, J. (2000). La investigación-acción en educación. (4a. ed.), Ediciones Morata, S. L.

Feo, R. (2010). Orientaciones básicas para el diseño de estrategias didácticas.

Guerrero, L. C. (2007). Normas, principios y estrategias comunicativas. Analecta malacitana.
Hymes, D. (2003). La competencia comunicativa.

MEN, C. (1998). Lineamientos Curriculares de Matemáticas. Magisterio, Bogotá.

MEN. (2006). Estándares básicos de competencias en Lenguaje, Matemáticas, Ciencias y Ciudadanas. (p. 15.)

Mosquera, F., \& Velasco, M. (2010). Estrategias didácticas para el aprendizaje colaborativo.

Obando, G., \& Vásquez, N. (2008). Pensamiento numérico del preescolar a la educación básica.

Piaget, J. (1950). Introducción a la Epistemología Genética. T1: El pensamiento matemático. T2: El pensamiento físico. T3: El pensamiento biológico, el pensamiento psicológico y el pensamiento sociológico. Buenos Aires: Paidós, Reeditado en 1975.

Saldaña, M. A. B. (2012). Apuntes teóricos sobre el pensamiento matemático y multiplicativo en los primeros niveles. Edma 0-6: Educación Matemática en la infancia, 1(1), 15-37.

Rodríguez Arocho, W. C. (1999). El legado de Vygotsky y de Piaget a la educación. Revista latinoamericana de psicología, 31(3). 